\title{
Impact of basic FGF expression in astrocytes on dopamine neuron synaptic function and development
}

\author{
Caroline Forget, ${ }^{1}$ Jane Stewart ${ }^{2}$ and Louis-Éric Trudeau ${ }^{1}$ \\ ${ }^{1}$ Department of Pharmacology, Faculty of Medicine, Université de Montréal, C.P. 6128, Succursale Centre-Ville Montréal, Québec, \\ Canada, H3C 3.7 \\ ${ }^{2}$ Center for Studies in Behavioural Neurobiology, Department of Psychology, Concordia University, Canada
}

Keywords: astrocytes, bFGF, dopamine release, rat, synapse formation

\begin{abstract}
Behavioural sensitization to amphetamine (AMPH) requires action of the drug in the ventral midbrain where dopamine (DA) neurons are located. In vivo studies suggest that AMPH sensitization requires enhanced expression of basic fibroblast growth factor (bFGF) in the nucleus of midbrain astrocytes. One idea is that the AMPH-induced increase in bFGF expression in astrocytes leads to enhanced secretion of this peptide and to long-term plasticity in DA neurons. To study directly the effects of astrocytic expression of bFGF on DA neurons, we established a cell-culture model of mesencephalic astrocytes and DA neurons. Immunolabelling showed that even in the absence of a pharmacological stimulus, the majority of mesencephalic astrocytes in culture express bFGF at a nuclear level. Arguing against the idea that bFGF was secreted, bFGF was undetectable in the extracellular medium (below $10 \mathrm{pg} / \mathrm{mL}$ ). However, supplementing culture medium with exogenous bFGF at standard concentrations $(20 \mathrm{ng} / \mathrm{mL})$ led to a dramatic change in the morphology of astrocytes, increased spontaneous DA release, and inhibited synapse formation by individual DA neurons. RNA interference (siRNA) against bFGF mRNA, caused a reduction in DA release but produced no change in synaptic development. Together these data demonstrate that under basal conditions (in the absence of a pharmacological stimulus such as amphetamine) bFGF is not secreted even though there is abundant nuclear expression in astrocytes. The effects of bFGF seen here on DA neurons are thus likely to be mediated through more indirect glial-neuronal interactions, leading to enhanced DA release without a necessary change in synapse number.
\end{abstract}

\section{Introduction}

Psychomotor stimulant drugs such as amphetamine (AMPH) produce multiple effects. Notable among them is their ability to increase extracellular levels of dopamine (DA), increase locomotor activity and support self-administration in humans and laboratory animals. The repeated intermittent administration of many drugs of abuse such as AMPH results in a progressive increase in their psychomotor activating and rewarding effects, a phenomenon known as behavioural sensitization (Segal \& Schuckit, 1983; Robinson \& Becker, 1986; Kalivas \& Stewart, 1991; Robinson \& Berridge, 1993; Vezina et al., 2002). One of the principal immediate actions of AMPH is to increase extracellular levels of DA in the terminal and cell body regions of midbrain DA neurons (Seiden et al., 1993; Sulzer et al., 1995; Kahlig et al., 2005).

Repeated administration of AMPH into the ventral tegmental area (VTA) but not into the nucleus accumbens (Nacc), is sufficient to induce sensitized behavioural responses and enhanced DA release in response to subsequent systemic injections of $\mathrm{AMPH}$, or cocaine (Kalivas \& Weber, 1988; Vezina \& Stewart, 1990; Vezina, 1993; Vezina, 1996). Glutamatergic transmission is necessary for sensitization. AMPH increases glutamate efflux in the VTA (Wolf \& Xue, 1998; Wolf \& Xue, 1999; Wolf et al., 2000) and both systemic and

Correspondence: Dr Louis-Éric Trudeau, as above.

E-mail: louis-eric.trudeau@umontreal.ca

Received 8 June 2005, revised 31 October 2005, accepted 26 November 2005
intra-VTA administration of NMDA, AMPA, or metabotropic glutamate receptors antagonists during the induction phase of sensitization prevent the development of sensitization to AMPH, or cocaine (Karler et al., 1989; Karler et al., 1990; Karler et al., 1991; Stewart \& Druhan, 1993; Li et al., 1997; Druhan \& Wilent, 1999; Li et al., 1999). The long-term changes induced in DA neurons by stimulant drugs might in addition result from the activation of growth factors, such as basic fibroblast growth factor (bFGF). Basic FGF is produced mainly by astrocytes and promotes growth and survival of midbrain DA cells (Chadi et al., 1993; Bouvier \& Mytilineou, 1995; Hou et al., 1997). Repeated intermittent AMPH administration increases bFGF immunoreactivity (IR) in the VTA and SN for up to one month (Flores et al., 1998). Moreover, the coadministration of AMPH and kynurenic acid, a broad-spectrum glutamate receptor antagonist, blocks the increase in bFGF-IR induced by AMPH. In addition, bFGF immunoneutralization blocks the induction of sensitization to AMPH and cotreatment with CPP, an NMDA receptor antagonist, prevents both the increase in the expression of bFGF in VTA and $\mathrm{SN}$ and the development of sensitization (Flores et al., 2000). Furthermore, we have found recently that AMPH induces dendritic growth in VTA DA neurons in vivo via endogenous bFGF (Mueller et al., 2006). Basic FGF in the VTA thus plays a critical role in sensitization to AMPH. However, whether bFGF is released or not from astrocytes is presently controversial (Abraham et al., 1986a; D'Amore, 1990).

The link between bFGF expression in VTA astrocytes and longterm plasticity in DA neurons is unclear. To begin addressing this 
question within a context that is not complicated by the multiples effects of AMPH in vivo, and that does not attempt to mimic sensitization, we used a primary mesencephalic culture system. We found abundant nuclear bFGF expression in mesencephalic astrocytes under basal condition but this was not accompanied by significant elevations of extracellular bFGF. Partial knockdown of bFGF using RNA interference caused a reduction in DA release but not in synapse number.

\section{Materials and methods \\ Cell culture}

For astrocyte cultures, neonatal (P0 to P2) Sprague-Dawley rats (Charles River, St-Laurent, QC, CAN) were cryoanaesthetized and their brains were rapidly removed and transferred into ice-cold dissociation solution, according to a protocol approved by the Université de Montréal animal ethics committee. A 1-mm-thick slice was cut at the level of the midbrain flexure from which a block of tissue, approximately $2 \mathrm{~mm}^{3}$ in size, was surgically isolated. The blocks were digested in papain for $60 \mathrm{~min}$ at $37^{\circ} \mathrm{C}$ before being gently triturated. The dissociated cells were diluted in Basal Medium Eagle (BME) and $5 \mathrm{~mL}$ of cell suspension was plated in flasks (two rats were used per flask). Forty-eight hours after plating, cells were washed with cold BME to kill neurons and select glial cells. After one week, glial cells were detached from the flask with a $0.05 \%$ trypsin solution and centrifuged. Cells were counted and plated on standard coverslips coated with collagen/poly L-lysine at 100000 cells $/ \mathrm{mL}$. When the cells reached confluence, after $48 \mathrm{~h}$, fluorodeoxyuridine (FUdR) was added to inhibit their proliferation.

Experiments measuring extracellular DA levels were performed using standard DA neuron cultures prepared according to recently described protocols (Bourque \& Trudeau, 2000; Bergevin et al., 2002; Congar et al., 2002; Jomphe et al., 2003), originally derived from Cardozo (1993) and Sulzer et al. (1998). Overall, the procedure involves plating neurons onto a pre-established monolayer of purified astrocytes. The coating and the layer of astrocytes partially mimic the extracellular matrix by promoting cell adhesion, growth, and survival. For some experiments, cultures were treated at day two and five after neuronal plating and used at day seven. For other experiments, cultures were treated at day five and seven and used at day ten after neuronal plating.

For neuronal cultures, neonatal (P0 to P2) Sprague-Dawley rats were cryoanaesthetized and their brains were rapidly removed and transferred into ice-cold dissociation solution. A 1-mm-thick slice was cut at the level of the midbrain flexure from which a block of tissue, approximately $2 \mathrm{~mm}^{3}$ in size, was surgically isolated. This block was found previously to contain most neuronal populations immunoreactive for tyrosine hydroxylase, a DA biosynthetic enzyme. The blocks were digested in papaïn for $30 \mathrm{~min}$ at $37^{\circ} \mathrm{C}$ before being gently triturated. The dissociated cells were collected by centrifugation, counted, and plated at a concentration of 350000 living cells per millilitre. This concentration gave consistent levels of extracellular DA and was optimal for neuronal viability. In order to provide an extracellular milieu as rich as possible in natural growth factors, a flask plated with astrocytes was filled with BME for two weeks to produce conditioned medium (BME+). Neuronal cultures were maintained in a solution composed of $(1: 3) \mathrm{BME}+$ and Neurobasal A supplemented with penicillin/streptomycin, GlutaMAX-1, 10\% fetal bovine serum (Gibco, USA) and B27 serum extender (Invitrogen). FUdR was added to the medium $24 \mathrm{~h}$ after neurons were plated to prevent cell division of newly plated cells, and kynurenic acid $(0.5 \mathrm{~mm})$, a broad-spectrum glutamate receptor antagonist, was added 7 days after plating in order to prevent excitotoxicity. Cell cultures were incubated at $37{ }^{\circ} \mathrm{C}$ in an atmosphere composed of $5 \% \mathrm{CO}_{2}$ and $95 \%$ air.

Experiments quantifying synaptic development were performed on isolated neurons in $\mu$-droplet cultures. In this model, cultured neurons grow on $\mu$-droplets of substrate that limit synaptic connectivity to the cells within the droplets. Under such conditions, single neurons establish synaptic connections onto themselves, called autapses, thus allowing measurements or quantification of synaptic development from single isolated neurons. Coverslips were first coated with poly L-ornithine and then agarose, which prevent cell adhesion. Collagen was applied on top of the agarose using a microsprayer, thus establishing droplets or islands of substrate that are suitable for cell growth. Astrocytes and neurons were then plated in much the same way as for standard cultures, although final concentrations for astrocytes and neurons were, respectively, 60000 and 100000 living cells per millilitres. These proportions maximized the number of $\mu$-droplets containing single neurons.

\section{Sampling procedure and dopamine assay}

In order to examine extracellular DA levels, standard neuronal cultures were deposited in a well containing $400 \mu \mathrm{L}$ of normal saline (NS) from which $100 \mu \mathrm{L}$ samples were drawn every $3 \mathrm{~min}$. The NS was composed of (in $\mathrm{mM}$ ): $\mathrm{NaCl} 140 ; \mathrm{KCl} \mathrm{5;} \mathrm{MgCl}_{2} 2 ; \mathrm{CaCl}_{2} 2$; sucrose 6; glucose 10; HEPES 10. Osmolarity was 305-310 mOsm and $\mathrm{pH}$ was adjusted to 7.35 with $\mathrm{NaOH} 5 \mathrm{M}$. Numbered aliquots were prepared prior to the experiment containing $2 \mu \mathrm{L}$ of preservative solution ( $\mathrm{pH}$ 6.5) consisting of ethylene glycol-bis( $\beta$-amino-ethyl ether)- $N, N, N^{\prime}, \mathrm{N}^{\prime}$-tetraacetic acid (EGTA, $90 \mathrm{mg} / \mathrm{mL}$ ) and reduced glutathion $(60 \mathrm{mg} / \mathrm{mL})$ in order to prevent the oxidation of DA. The aliquots also contained $20 \mu \mathrm{L}$ of distilled water to yield sufficient volume for the automatic injection into the HPLC system (automatic injection of $100 \mu \mathrm{L}$ ). The data shown in this paper were corrected for this dilution. The collected $100 \mu \mathrm{L}$-samples of extracellular medium ( $25 \%$ of total volume) withdrawn from the well were dropped into the numbered aliquots and immediately replaced with an equivalent volume of fresh NS. The aliquots were put on dry ice for the duration of the experiment (usually $50 \mathrm{~min}$ ) and then stored at $-80{ }^{\circ} \mathrm{C}$ until analysis with the HPLC system. The first four aliquots were usually not analysed as preliminary experiments showed that extracellular levels of DA required 15 min to stabilize. Samples were thawed and then placed onto the stage of the HPLC system (Gilson, Villiers-LeBel, France), to be injected automatically every $10 \mathrm{~min}$. HPLC coupled to a coulometric detector (Coulochem II; ESA, Bedford, USA) was used to detect DA (detection limit approximately $25 \mathrm{pg} / 100 \mu \mathrm{L}$ sample). For experiments evaluating the impact of treatments on evoked DA release, saline containing $40 \mathrm{~mm}$ potassium was used. $\mathrm{NaCl}$ was lowered by $40 \mathrm{mM}$ to prevent changes in osmolarity.

\section{Immunocytochemistry}

Cells were fixed with $100 \%$ methanol for $5 \mathrm{~min}$ at $4{ }^{\circ} \mathrm{C}$, permeabilized with $0.1 \%$ Triton $\mathrm{X}-100$ for $10 \mathrm{~min}$ and bathed for $5 \mathrm{~min}$ in a solution containing BSA $(0.5 \%)$ in order to block nonspecific binding sites. Cells were then incubated overnight at $4{ }^{\circ} \mathrm{C}$ with primary antibodies; rabbit monoclonal antibody against TH (1: 1000; Pelfrez Biological, USA), mouse monoclonal anti bFGF (1 : 500; Upstate biotechnology, Lake Placid, NY), mouse monoclonal anti-SV2 (1 : 500; Developmental Studies Hybridoma Bank, Iowa, USA) or 
rabbit polyclonal anti-cow S-100 (1 : 5000; DAKO, California, USA). Primary antibodies were detected using Alexa-488 or Alexa-647 (1 : 200; Molecular Probes Inc., Eugene, OR) fluorescently labelled secondary antibodies before being finally mounted with Vectashield (Vector Laboratories, Burlingame, USA).

\section{Confocal microscopy}

$\mu$-droplet neuronal cultures pretreated with neutralizing anti-bFGF antibody, exogenous bFGF or siRNA against bFGF were immunolabelled with anti-TH and anti-SV2. Primary antibodies were detected using Alexa-488 and Alexa-647, respectively. Images were acquired using a point-scanning confocal microscope from Prairie Technologies LLC (Middleton, WI, USA). Excitation was achieved using the $488 \mathrm{~nm}$ line of an argon ion laser and the $633 \mathrm{~nm}$ line of a helium neon $(\mathrm{HeNe})$ laser. Images were analysed using Metamorph software v4.5 from Universal Imaging Corp (Downingtown, PA, USA).

\section{Small interfering RNA (siRNA)}

The siRNA were synthesized in vitro with a construction kit from Ambion (Austin, TX, USA). Three different oligonucleotides were tested and all three were effective at reducing bFGF levels, although to a different extent. The sequence of the first siRNA was AAGGGAGAAAGTTGCATTTAACCTGTCTC (antisens) and AATTAAATGCAACTTTCTCCCCCTGTCTC (sens); it reduced bFGF levels by $40 \%$ (results not shown). The sequence of the second siRNA (siRNA \#2) was AAGAGTGTTTCTTCTTTGAACCCTGTCTC (antisens) and AAGTTCAAAGAAACACTCCCTGTCTC (sens); it reduced bFGF levels by $60 \%$. The sequence of the third siRNA (siRNA \#3) was AAACGAGGGCAGTATAAACCTGTCTC (antisens) and AATTTATACTGCCCAGTTCGTCCTGTCTC (sens); it reduced bFGF levels by $30 \%$ (results not shown). The second was thus selected for further experimentation in which we measured the impact of the siRNA on extracellular DA levels and on synapse number. Control experiments were performed with siRNA against glyceraldehyde-3-phosphate dehydrogenase (GAPDH) and without any siRNA. RNAiFect reagent from Qiagen (Mississauga, Ontario, CAN) was used for lipofection. Depending on the cell surface the quantity of siRNA and RNAifect reagent were changed respecting a $1: 5$ ratio ( $1 \mu \mathrm{g}$ of siRNA for $5 \mu \mathrm{L}$ of RNAifect reagent). The astrocytes were incubated for $24 \mathrm{~h}$ with the transfection complex.

\section{Western blot}

Purified astrocytes from midbrain primary cultures were transfected with siRNA against bFGF, using RNAiFect lipofection reagent (Qiagen). The transfection complexes were applied to cells for $24 \mathrm{~h}$. Cell monolayers were then washed with Tris-buffered saline (TBS; $\mathrm{pH} 7.4$ ) and immediately lysed (lysis buffer; TBS with $1 \%$ of IGEPAL CA-630 and $0.5 \%$ SDS $20 \%$ with added protease inhibitors; PMSF 1:100, leupeptin 1:100 and pepstatin $1: 1000)$. Cell lysates $(30 \mu \mathrm{g})$ were resolved by electrophoresis on $15 \%$ polyacrylamide minigels and transferred on pure nitrocellulose membrane $(0.45 \mu \mathrm{m})$. Blots were probed with anti-bFGF ( $1: 500)$ and anti- $\beta \gamma$-actin $(1: 10$ 000; Abcam, Cambridge, MA, USA). Bound antibodies were detected using horseradish peroxidase-conjugated secondary antibodies (Jackson immunoresearch laboratories, West Grove, PA, USA) and revealed by chemiluminescence (SuperSignal, West Pico Chemoluminescence Substrate, Pierce, Rockford, IL, USA). The blots were quantified by densitometric evaluation with ImageQuant software (Amersham Biosciences, Baie d'Urfé, Qc, CAN).

\section{ELISA test against bFGF}

A human basic FGF ELISA Kit from Calbiochem (San Diego, CA, USA) was used to evaluate the extracellular concentration of bFGF. We first performed standard curves with bFGF protein at known concentrations $(160,80,40,20,10$ and $0 \mathrm{pg} / \mathrm{mL})$. Optical density was recorded for the standard samples and unknown samples and the bFGF concentration was calculated according to the standard curve.

\section{Results \\ bFGF is expressed in the nucleus of the majority of cultured mesencephalic astrocytes}

We first examined the expression of bFGF in primary mesencephalic cells in culture. Using double-label immunocytochemistry to detect bFGF and the astrocyte-specific protein S-100, we found that bFGF immunoreactivity was present in the majority of astrocytes but not in neurons (Fig. 1A-D). The labelling was essentially nuclear in all cells examined (Fig. 1E). Western blots prepared from purified astrocytes showed that all three bFGF isoforms can be detected (Fig. 1F).

\section{bFGF does not appear to be secreted}

The capacity of bFGF-expressing cells to release this growth factor is controversial. We thus performed an ELISA test to determine whether the high expression of bFGF by mesencephalic astrocytes is accompanied by detectable extracellular accumulation of bFGF. Culture medium from astrocyte cultures, astrocyte-neuron mixed cultures or control medium not exposed to cells were compared. In all cases, bFGF levels were found to be below the smallest value included in our standard curve, which was $10 \mathrm{pg} / \mathrm{mL}$. The values measured from culture mediums taken from astrocyte cultures were similar to those measured from fresh mediums not exposed to astrocytes and were thus considered to reflect assay noise (Fig. 2A). These data show that abundant bFGF expression by cultured mesencephalic astrocytes is not accompanied by significant secretion of this polypeptide.

\section{Additional support for a lack of bFGF secretion}

In support of our conclusion of a lack of bFGF secretion, we found that the morphology of cultured astrocytes in our model did not show the characteristic highly stellar shape of astrocytes exposed to bFGF (Eclancher et al., 1996a; Reilly et al., 1998; Brambilla et al., 2003). Indeed, treatment of cultured astrocytes with exogenous bFGF produced the expected striking alteration in cell morphology (Fig. 2B and C).

Another prediction deriving from the lack of bFGF secretion is that extracellular application of a bFGF function-blocking antibody should fail to affect DA neurons. Using isolated DA neurons after two days in $\mu$-culture (Fig. 3A), we quantified synaptic development following a five-day treatment with neutralizing anti-bFGF mouse monoclonal antibody $(10 \mu \mathrm{g} / \mathrm{mL})$. We found that the treatment had no effect on the synaptic development of DA neurons (Fig. 3B). As a positive control, we also examined the effect of exogenous bFGF $(20 \mathrm{ng} / \mathrm{mL})$ 
A

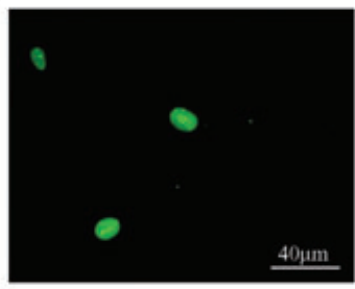

B

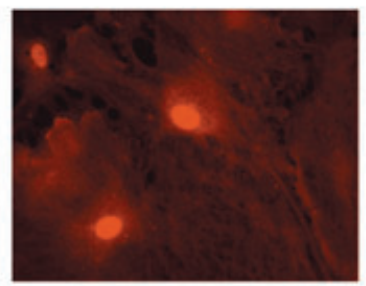

C

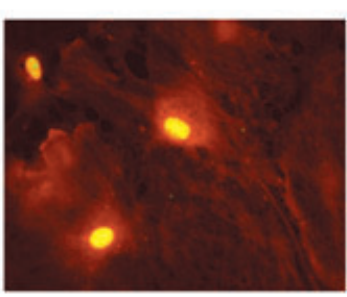

E

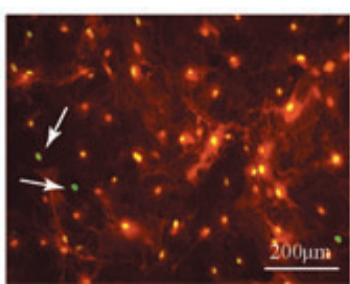

D

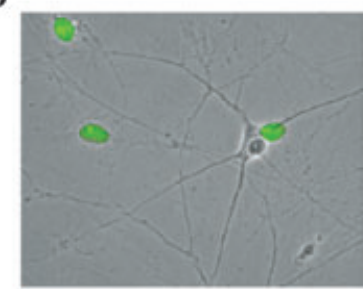

F
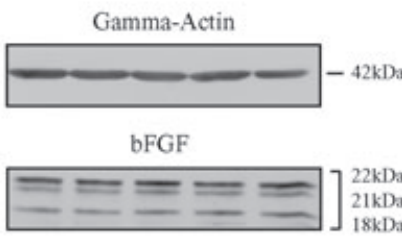

FIG. 1. bFGF is expressed in the majority of cultured mesencephalic astrocytes. (A) Immunofluorescence image showing nuclear expression of bFGF in mesencephalic astrocytes in culture (green). (B) Same astrocytes as in A after immunostaining against the glial protein S-100 (red). (C) Colocalization (yellow) of bFGF (green) and S-100 (red) shows that bFGF is expressed in the nucleus of astrocytes. (D) Phase contrast image merged with bFGF immunoreactivity (green) showing astrocytes and neurons in co-culture. Note that only astrocytes show bFGF immunolabelling. (E) bFGF and S-100 double-labelling examined at low power $(10 \times)$. Note that the vast majority of astrocytes (red) express bFGF (green). The white arrows identify some of the few cells that were bFGF-positive but S-100-negative. (F) Western blot analysis, of multiple extracts prepared from mesencephalic astrocytes, showing that the three bFGF isoforms $(18,21$ and $22 \mathrm{kDa})$ are expressed. Gamma actin (42 kDa) was used as loading control.

A

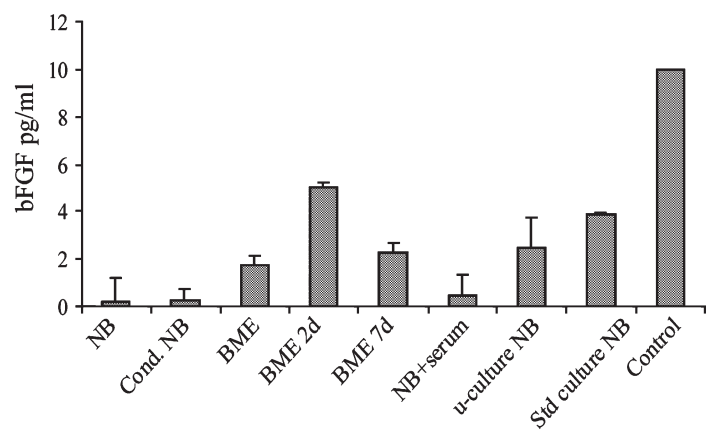

B
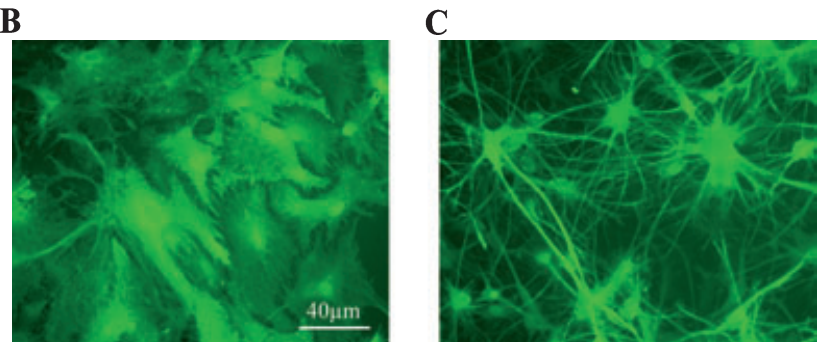

A
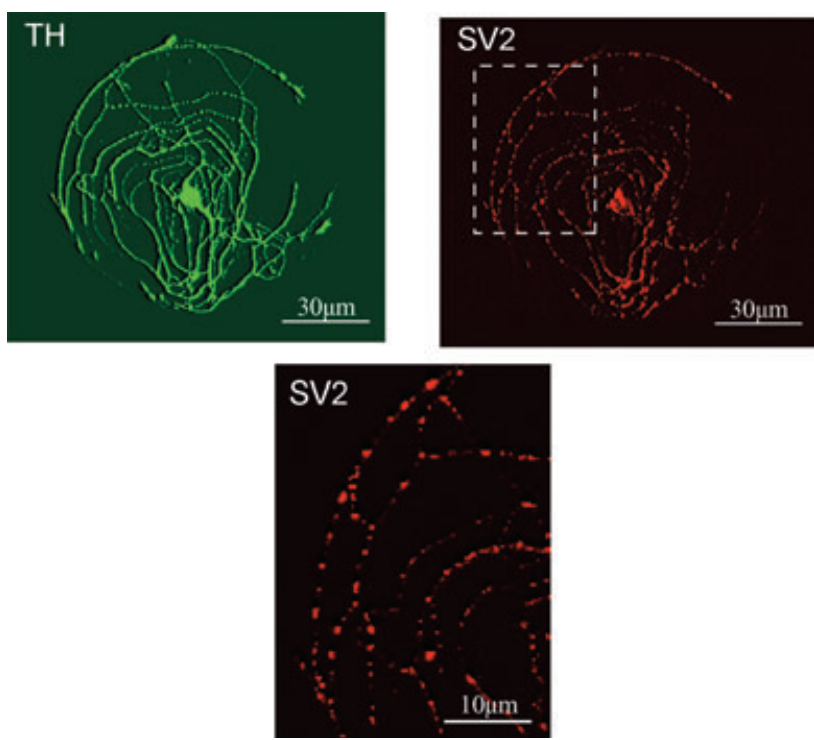

B

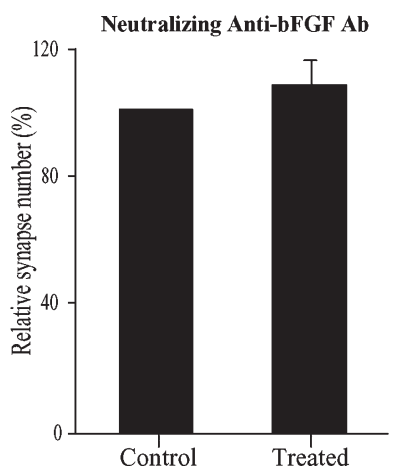

C

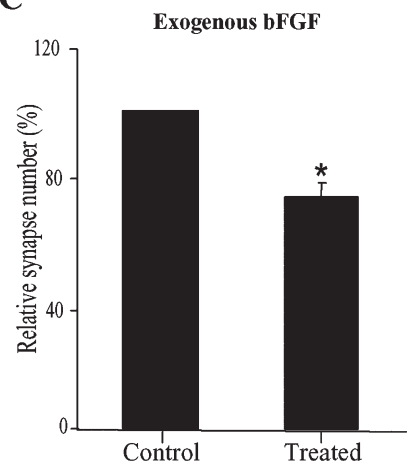

FIG. 3. Extracellular bFGF does not increase synaptic development. (A) Single neurons in $\mu$-culture after immunostaining for tyrosine hydroxylase (TH) (green) and for the synaptic vesicle protein SV2 (red). The lower image shows a higher resolution portion of the SV2 signal delimited by the white dotted box. Small SV2+ punctate structures were considered as putative axon terminals and counted using Metamorph Software. (B) Five days treatment with a bFGF neutralizing antibody $(10 \mu \mathrm{g} / \mathrm{mL})$ did not change the number of morphologically identifiable axon terminals counted at day seven in culture $(n=44)$ compared to control $(n=49)$ ( $t$-test, $P>0.05)$. (C) Five days treatment with exogenous bFGF $(20 \mathrm{ng} / \mathrm{mL})$ caused a decrease in synapse number, counted at day seven in culture $(n=32)$ compared to control $(n=27)$ $\left(t\right.$-test, $\left.{ }^{*} P<0.05\right)$.

FIG. 2. bFGF does not appear to be secreted. (A) A bFGF ELISA test was performed with different culture media including: fresh neurobasal without serum (NB), neurobasal conditioned by exposure to neurons and astrocytes (Cond. NB), fresh basal medium eagle (BME), BME conditioned by exposure to purified astrocytes grown in flask for 2 days (BME 2d) or 7 days (BME 7d), neurobasal with serum (NB + serum), and neurobasal conditioned by exposure to neurons and astrocytes in microculture ( $\mu$-culture NB) and standard cultures (Std culture NB). Finally, the control column represents the signal produced by the lowest tested concentration of bFGF measured with the ELISA test $(10 \mathrm{pg} / \mathrm{mL})$ and represents the limit of detection. Note that in all cases, bFGF concentrations were below the level of detection. (B) Immuno-labelling against S-100 protein in a control culture. (C) Immuno-labelling against S-100 protein in a culture treated for 7 days with exogenous bFGF $(20 \mathrm{ng} / \mathrm{mL})$. Note the stellate appearance of astrocytes after treatment with bFGF. 
on synaptic development. As shown in Fig. 3C, exogenous bFGF actually caused a small but significant inhibition of synaptic development.

To provide a more direct evaluation of the functioning of DA neurons, we also used HPLC to measure basal as well as $\mathrm{K}^{+}$-evoked DA levels in standard mixed mesencephalic cultures. Each coverslip contained between 300 and 800 spontaneously firing DA neurons (St-Gelais et al., 2004). We initially treated standard cultures at day two in culture with exogenous bFGF $(20 \mathrm{ng} / \mathrm{mL})$, repeating the treatment at day four in culture. DA levels were then sampled at day seven. Exposure to bFGF caused a significant increase in the basal level of extracellular DA $(197.35 \pm 8.80 \mathrm{pg} / \mathrm{mL}, n=21)$ compared to control cells not exposed to bFGF $(149.36 \pm 7.15 \mathrm{pg} / \mathrm{mL}, n=15$; $P<0.05$; Fig. 4A). Potassium-evoked DA release was not significantly affected by exogenous bFGF $(768.00 \pm 71 \mathrm{pg} / \mathrm{mL}, n=21 \mathrm{vs}$. $710.40 \pm 80 \mathrm{pg} / \mathrm{mL}, n=15$; Fig. 4B). Similar experiments were performed on more mature cultures treated at days five and seven in culture, with DA sampled at day ten. Basal DA release was again significantly higher after bFGF treatment $(351.92 \pm 37.3 \mathrm{pg} / \mathrm{mL}$, $n=18$ vs. $236.13 \pm 24 \mathrm{pg} / \mathrm{mL}, n=12$; $P<0.05$; Fig. 4C). Potassium-evoked DA release showed a modest but nonsignificant increase $(1954.61 \pm 245 \mathrm{pg} / \mathrm{mL}, n=18$ vs. $1574.22 \pm 203 \mathrm{pg} / \mathrm{mL}, n=12$; Fig. 4D). Together these findings show that exogenous bFGF can affect the function of DA neurons and the morphology of astrocytes. These findings argue that if endogenous bFGF had been released by astrocytes in our cultures, it should have produced effects similar to those produced by exogenous bFGF and these effects should have been blocked by a function-blocking antibody. The effects of this growth factor on DA neurons are therefore unlikely to be mediated through extracellular secretion.
RNA interference against bFGF decreases dopamine release but not synapse number

If bFGF is not secreted, an alternate hypothesis is that it acts within astrocytes to indirectly affect the functioning of DA neurons. To test this hypothesis we used RNA interference (siRNA \#2) to decrease endogenous levels of bFGF in confluent cultured astrocytes. Figure 5A and $\mathrm{B}$ shows that bFGF expression was decreased by $50-60 \% 24 \mathrm{~h}$ after transfection with siRNAs. Quantified independently, the $18 \mathrm{kDa}$ isoform was decreased to $48.58 \pm 8.60 \%$ of control $(n=5$; $P<0.05)$, the $21 \mathrm{kDa}$ isoform to $40.35 \pm 9.98 \%$ of control $(n=5$; $P<0.05)$ and the $22 \mathrm{kDa}$ isoform to $43.78 \pm 8.54 \%$ of control $(n=5)$. Transfection with a control siRNA directed against GAPDH failed to significantly affect bFGF levels (Fig. 5A). Additional experiments were performed with two other siRNAs with different sequences (siRNA \#1 and siRNA \#3; see Materials and methods section). These were also effective at decreasing bFGF levels, but the effect was smaller $(40 \%$ and $30 \%$ decrease, respectively; results not shown).

Using standard mixed cultures we next transfected siRNA against bFGF at day seven in culture and sampled extracellular DA levels at day ten in culture. Down regulation of bFGF in astrocytes caused a significant reduction in basal DA levels $(115.22 \pm 7.31 \mathrm{pg} / \mathrm{mL}$, $n=14$, vs. $161.57 \pm 20.60 \mathrm{pg} / \mathrm{mL}, n=9 ; P<0.05$; Fig. 6A). Potassium-evoked DA release was also slightly decreased, but the effect did not reach statistical significance $(1012.12 \pm 111.4 \mathrm{pg} / \mathrm{mL}$, $n=14$, vs. $1346.12 \pm 161.0 \mathrm{pg} / \mathrm{mL}, n=9$; Fig. 6B). Separate experiments were performed with a second siRNA of a different sequence (siRNA \#1). As expected because of its lower efficacy at decreasing bFGF levels, it failed to significantly reduce basal or potassium-evoked DA release (results not shown).
$\mathbf{A}$

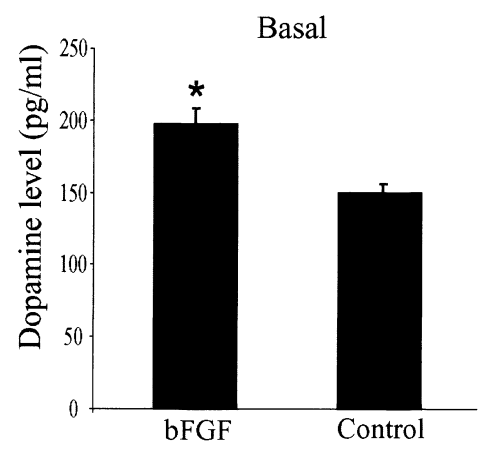

C

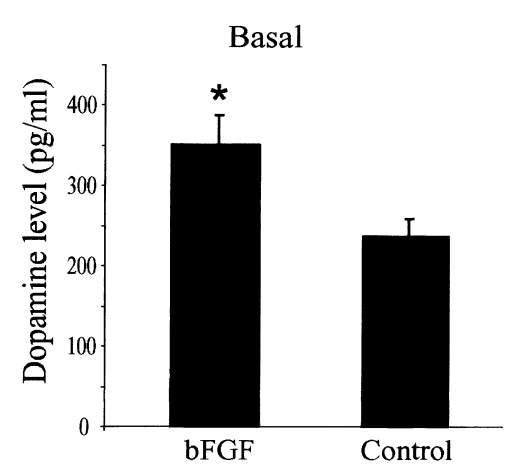

B

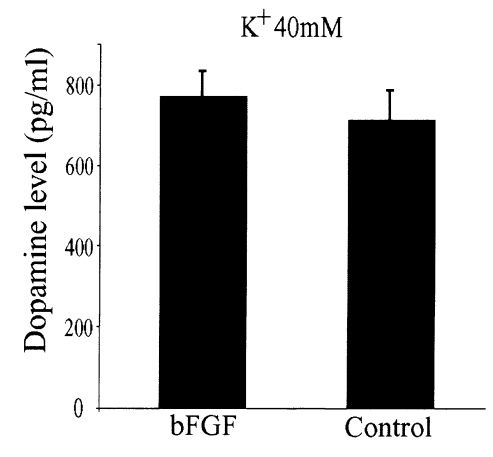

D

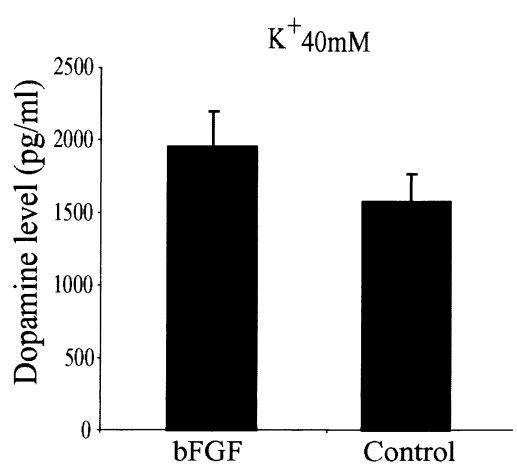

FIG. 4. Exogenous bFGF enhances dopamine release. HPLC was used to measure basal (due to spontaneous firing) and $40 \mathrm{mM} \mathrm{K}^{+}$evoked DA release. bFGF was applied at days two and four in culture, and DA sampling was performed at day seven in culture. (A) Average DA concentrations during the baseline period. Basal levels were significantly higher after exogenous bFGF $(20 \mathrm{ng} / \mathrm{mL})(n=21)$ in comparison to control $(n=15)(t$-test, $* P<0.05)$. (B) Average DA concentrations during the $40 \mathrm{mM} \mathrm{K}{ }^{+}$stimulation. Stimulated DA levels were not significantly higher after exogenous bFGF $(20 \mathrm{ng} / \mathrm{mL})$ in comparison to control ( $t$-test, $P>0.05)$. (C and D) Same experiment as in A except that bFGF was applied at days five and seven in culture and sampling were performed at day ten. Note that DA levels are higher in these cultures due to more extensive synaptic development after 10 days in culture. Basal levels were again significantly higher after exogenous bFGF $(20 \mathrm{ng} / \mathrm{mL})(n=18)(t$-test, $* P<0.05)$ compared to control $(n=12)$. Stimulated DA levels were again not significantly higher after exogenous bFGF $(20 \mathrm{ng} / \mathrm{mL})$ in comparison to control ( $t$-test, $* P>0.05)$. 
A

\section{Gamma-Actin}

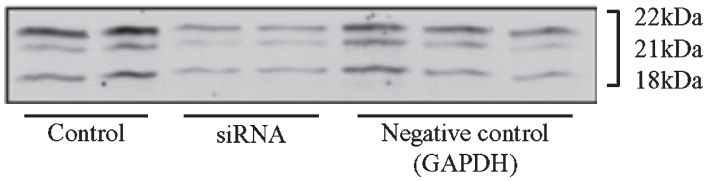

B

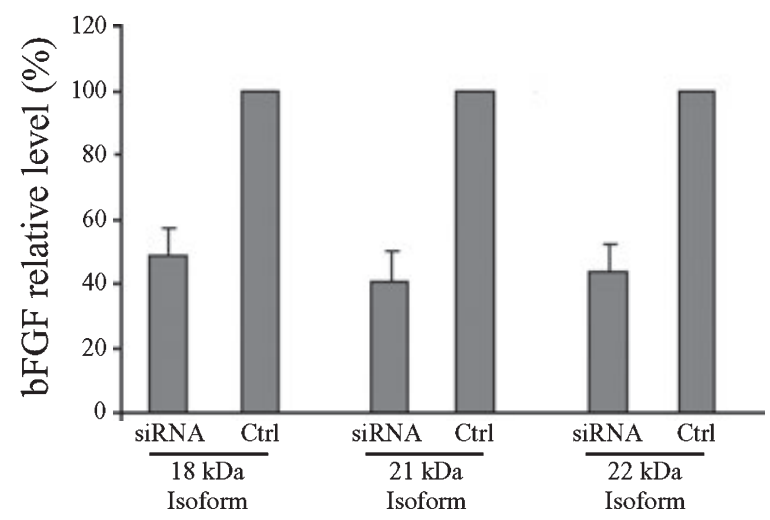

FIG. 5. RNA interference decreases bFGF levels. (A) Western blot showing the decrease of the three bFGF isoforms (18, 21 and $22 \mathrm{kDa})$ after transfection with siRNA against bFGF mRNA compared to control (nontransfected cells), $n=5$. Used as a negative control, siRNA against GAPDH did not reduce bFGF levels. Gamma actin $(42 \mathrm{kDa})$ was used as a loading control. (B) Histogram showing the quantification of bFGF isoforms after siRNA transfection, $t$-test, $* P<0.05$.

FIG. 6. bFGF siRNA decreases dopamine release but not synapse number. HPLC was used to measure basal (due to spontaneous firing) and $40 \mathrm{mM} \mathrm{K}^{+}$evoked DA release. Control cells $(n=9)$ and siRNA transfected cells $(n=14)$ were treated at day seven in culture and sampling was performed at day ten in culture. (A) Average DA concentrations during the baseline period. siRNA treatment caused a significant decrease relative to the control group ( $t$-test, $P<0.05$ ).

(B) Average DA concentrations during the $40 \mathrm{mM} \mathrm{K}^{+}$stimulation. Stimulated DA levels were not significantly decreased by siRNA treatment relative to the control group ( $t$-test, $P>0.05$ ).

(C) Single neuron cultures transfected with siRNA $(n=97)$ did not show a decrease in synapse number at day seven in culture compared to control $(n=65)$.
$\mathbf{A}$

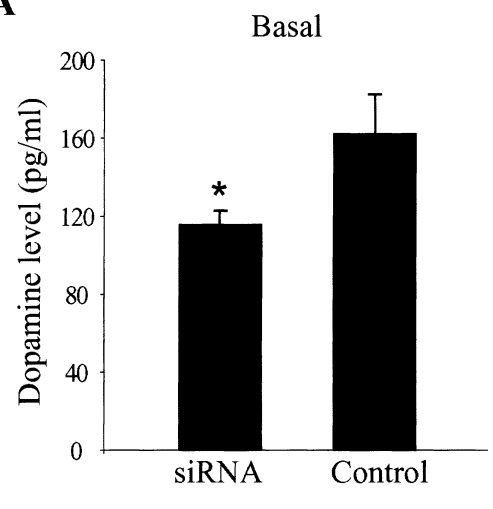

B

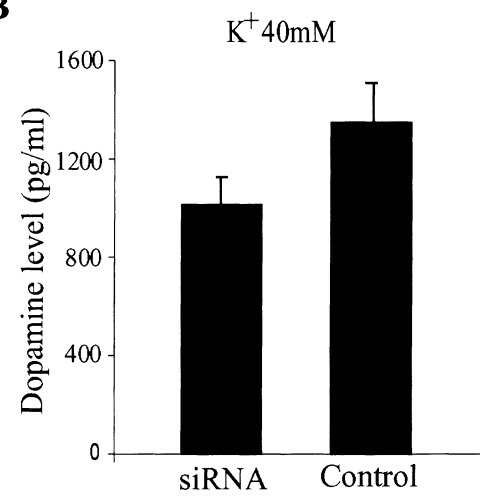

siRNA transfection experiments were also performed (siRNA\#2) in isolated DA neuron at day two in culture and fixed for analysis at day seven in culture. As shown in Fig. 6C, this failed to affect synaptic development. It would appear from these findings that a reduction of bFGF protein levels in astrocytes reduces basal DA release, but does not affect the synaptic development of DA neurons.

\section{Discussion}

The objective of the present study was to establish the role of astrocytic bFGF in the synaptic development and function of DA neurons. We show that in VTA astrocytes in primary culture, bFGF

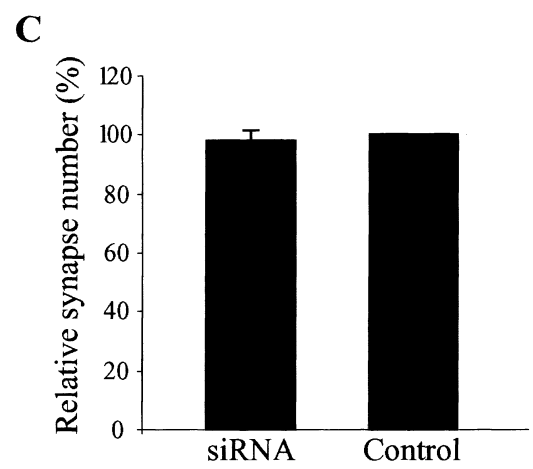

protein is expressed in the vast majority of cells. However, its expression is exclusively nuclear and it does not appear to be actively secreted. Using a RNA interference strategy, we found that decreasing endogenous bFGF protein levels produced no change in synapse number, but decreased DA release. Taken together, these results suggest that astrocytic bFGF acts indirectly on DA neurons to enhance their synaptic function without directly influencing morphological synapse development.

Although this has never been quantified directly, it appears that in the mature brain, only a relatively small proportion of astrocytes express nuclear bFGF under basal conditions (Tessler \& Neufeld, 1990; Woodward et al., 1992; Szele et al., 1995; Salik et al., 2005). 
Interestingly, the number of astrocytes that express nuclear bFGF in the ventral midbrain increases following repeated-intermittent AMPH administration (Flores et al., 1998; Flores et al., 1999; Flores et al., 2000). As we find that most cultured astrocytes express nuclear bFGF, it may be that the process of cell dissociation and culture puts these cells in a state similar to that found after sensitization to drugs of abuse (Flores et al., 1998).

Basic FGF is a polypeptide that exists in three isoforms with molecular weights of 18,21 and $22 \mathrm{kDa}$, respectively, in the rat (Powell \& Klagsbrun, 1991; Giordano et al., 1992). The $18 \mathrm{kDa}$ isoform is reported to be predominantly cytoplasmic while the high molecular weight isoforms are preferentially localized in nuclear and ribosomal compartments (Renko et al., 1990; Florkiewicz et al., 1991; Klein et al., 1996). Even if all three isoforms are expressed in our preparation, as demonstrated by Western blotting, only nuclear expression could be detected in cultured mesencephalic astrocytes using immunocytochemistry (Fig. 1). Human astrocytes have however, been reported to express both nuclear and cytoplasmic bFGF (Stachowiak et al., 1994; Moffett et al., 1996; Joy et al., 1997). The capacity of bFGF to be secreted has long been the subject of debate. The mechanism of bFGF secretion remains unclear as the peptide bears no signal sequence (Abraham et al., 1986a). bFGF also does not progress through the endoplasmic reticulum and the Golgi via the regulated secretory pathway (Abraham et al., 1986a; Abraham et al., 1986b; Schweigerer et al., 1987). It has been suggested that bFGF is released from cells as the result of cell death or cell damage, during nonlethal membrane disruption or following chemical injuries (Klagsbrun \& Vlodavsky, 1988; McNeil et al., 1989; D’Amore, 1990; Ku \& D'Amore, 1995). Others have proposed that bFGF is released by cells via a secretory pathway that is independent from the endoplasmic reticulum or Golgi (Vlodavsky et al., 1987; D'Amore, 1990; Rifkin et al., 1991; Mignatti et al., 1992; Florkiewicz et al., 1995). Moreover, Le Roux \& Esquenazi (2002), used Western blotting to demonstrate the presence of bFGF in media conditioned by cortical astrocytes. Although we have not evaluated here whether the bFGF contained in our astrocytes can be released by chemical or pathological stimuli, our ELISA measurements show quite clearly that extracellular bFGF levels are extremely low and actually correspond to background signal (Fig. 2A). Such low levels are insufficient to produce effects typically induced by modest concentrations of exogenous bFGF such as induction of a stellate morphology in astrocytes (Eclancher et al., 1990; Eclancher et al., 1996b; Reilly et al., 1998), an effect that was readily seen in our experiments after short-term exposure to exogenous bFGF (Fig. 2C). Compatible with the hypothesis that bFGF is not released, we observed that treatment of isolated DA neurons with bFGF neutralizing antibodies did not change the synaptic development of DA neurons (Fig. 3A and B). However, exogenous bFGF caused a modest but significant decrease in synapse number (Fig. 3C). This observation is compatible with the earlier work of Williams et al. (1995), who showed that bFGF can under certain circumstances inhibit neurite outgrowth in a dose dependent manner via arachidonic acid production (Williams et al., 1995). We thus conclude that the abundant nuclear bFGF is not released in any significant manner in our midbrain astrocytes cultures.

Even though we found that bFGF is not released, a decrease in endogenous bFGF levels in response to RNAi directed against bFGF mRNA caused a decrease in DA release (Fig. 6A). This implies that astrocytic bFGF acts to promote DA release. If bFGF is not released from astrocytes, then how could it lead to plastic changes in DAergic neurons such as enhanced DA release? Trudel et al. (2000) proposed that bFGF can be translocated to the cell surface without release in the conditioned media. This hypothesis is not readily compatible with our results as whether it is released or not in the medium, its presence on the extracellular membrane should activate bFGF receptors and cause physiological effects such as induction of a stellate morphology in astrocytes. Another interesting hypothesis proposed by Stachowiak and collaborators (Stachowiak et al., 1997a; Stachowiak et al., 1997b; Hu et al., 2004) is that FGFR1 is localized in the nucleus and could serve as an effector for nuclear bFGF. Cellular effects of bFGF could indeed be mediated by an intracellular (intracrine) pathway (Logan, 1990; Sherman et al., 1993; Bouche et al., 1987; Nakanishi et al., 1992; Arese et al., 1999) that operates through nuclear FGFR1 and perhaps through transcription factors such as c-jun (Hortala et al., 2005). Functional FGFR1 within the nucleus could mediate the mitogenic effects of bFGF in many cell types (Imamura et al., 1990; Wiedlocha et al., 1994; Bikfalvi et al., 1995; Joy et al., 1997). Activation of such intracrine signal pathways in astrocytes could thus lead to changes in the expression of astrocytes-derived growth factors such as GDNF, that are well-known to influence the development of DA neurons as well as their capacity to release DA (Bourque \& Trudeau, 2000; Feng et al., 1999). Although this has not yet been examined, nuclear bFGF could also perhaps regulate the release of gliotransmitters such as glutamate or ATP that could subsequently act on DA neurons (Cotrina et al., 2000; Haydon, 2001; Ullian et al., 2004).

Based on our results, it may now be necessary to reconsider the range of possible models proposed by Flores \& Stewart (2000b) to explain AMPH sensitization. As previously proposed, AMPH may lead to $\mathrm{D}_{1}$ DA receptor stimulation in the VTA (Hosli \& Hosli, 1993; Bal et al., 1994; Luo et al., 1998; Zanassi et al., 1999), thus causing en elevation of extracellular glutamate and stimulation of bFGF synthesis in astrocytes. We can now hypothesize that at least in part, some of the long-term plasticity induced in DA neurons under such circumstances (Mueller et al., 2006) may result from bFGF-mediated intracrine signalling in astrocytes, leading to the generation of a secondary diffusible messenger or some contact-dependent signal in DA neurons. Further experiments will be required to test this hypothesis and evaluate more closely the nature of the glial-neuronal mechanisms potentially involved. Another open question that should be addressed is whether the ability of astrocytes to regulate DA release through bFGF requires glial-neuronal interactions selectively at the somatodendritic level or if similar effects can be mediated by astrocytes at the terminal level in structures such as the striatum. Such an issue would not be readily studied in a cell culture system where DAergic axons and cell bodies are in close proximity.

In conclusion, our work shows that cultured mesencephalic astrocytes express abundant levels of bFGF in their nuclear compartment but that this growth factor may not necessarily be released. Instead, intracellular activation of bFGF signalling pathways may regulate astrocyte function and signals, indirectly leading to the functional regulation of DA neurons.

\section{Acknowledgements}

This work was supported by the National Science and Engineering Research Council of Canada (NSERC) and in part by a team grant from Fonds de Recherche sur la Nature et les Technologies (Québec). Louis-Éric Trudeau is a scholar of the Fonds de la Recherche en Santé du Québec. We wish to thank Marie-Josée Bourque for her important contribution to the preparation and maintenance of the neuronal cultures and for image capture and processing.

\section{Abbreviations}

AMPH, amphetamine; bFGF, basic fibroblast growth factor; BME, Basal Medium Eagle; DA, dopamine; NS, normal saline; siRNA, small interfering RNA; VTA, ventral tegmental area. 


\section{References}

Abraham, J.A., Mergia, A., Whang, J.L., Tumolo, A., Friedman, J., Hjerrild, K.A., Gospodarowicz, D. \& Fiddes, J.C. (1986a) Nucleotide sequence of a bovine clone encoding the angiogenic protein, basic fibroblast growth factor. Science, 233, 545-548.

Abraham, J.A., Whang, J.L., Tumolo, A., Mergia, A., Friedman, J., Gospodarowicz, D. \& Fiddes, J.C. (1986b) Human basic fibroblast growth factor: nucleotide sequence and genomic organization. EMBO J., 5, 25232528.

Arese, M., Chen, Y., Florkiewicz, R.Z., Gualandris, A., Shen, B. \& Rifkin, D.B. (1999) Nuclear activities of basic fibroblast growth factor: potentiation of low-serum growth mediated by natural or chimeric nuclear localization signals. Mol. Biol. Cell, 10, 1429-1444.

Bal, A., Bachelot, T., Savasta, M., Manier, M., Verna, J.M., Benabid, A.L. \& Feuerstein, C. (1994) Evidence for dopamine D2 receptor mRNA expression by striatal astrocytes in culture: in situ hybridization and polymerase chain reaction studies. Brain Res. Mol. Brain Res., 23, 204-212.

Bergevin, A., Girardot, D., Bourque, M.J. \& Trudeau, L.E. (2002) Presynaptic mu-opioid receptors regulate a late step of the secretory process in rat ventral tegmental area GABAergic neurons. Neuropharmacology, 42, 1065-1078.

Bikfalvi, A., Klein, S., Pintucci, G., Quarto, N., Mignatti, P. \& Rifkin, D.B. (1995) Differential modulation of cell phenotype by different molecular weight forms of basic fibroblast growth factor: possible intracellular signaling by the high molecular weight forms. J. Cell Biol., 129, 233243.

Bouche, G., Gas, N., Prats, H., Baldin, V., Tauber, J.P., Teissie, J. \& Amalric, F. (1987) Basic fibroblast growth factor enters the nucleolus and stimulates the transcription of ribosomal genes in ABAE cells undergoing G0-G1 transition. Proc. Natl Acad. Sci. USA, 84, 6770-6774.

Bourque, M.J. \& Trudeau, L.E. (2000) GDNF enhances the synaptic efficacy of dopaminergic neurons in culture. Eur. J. Neurosci., 12, 3172-3180.

Bouvier, M.M. \& Mytilineou, C. (1995) Basic fibroblast growth factor increases division and delays differentiation of dopamine precursors in vitro. J. Neurosci., 15, 7141-7149.

Brambilla, R., Cottini, L., Fumagalli, M., Ceruti, S. \& Abbracchio, M.P. (2003) Blockade of A2A adenosine receptors prevents basic fibroblast growth factor-induced reactive astrogliosis in rat striatal primary astrocytes. Glia, 43, $190-194$.

Cardozo, D.L. (1993) Midbrain dopaminergic neurons from postnatal rat in long-term primary culture. Neuroscience, 56, 409-421.

Chadi, G., Moller, A., Rosen, L., Janson, A.M., Agnati, L.A., Goldstein, M., Ogren, S.O., Pettersson, R.F. \& Fuxe, K. (1993) Protective actions of human recombinant basic fibroblast growth factor on MPTP-lesioned nigrostriatal dopamine neurons after intraventricular infusion. Exp. Brain Res., 97, 145158.

Congar, P., Bergevin, A. \& Trudeau, L.E. (2002) D2 receptors inhibit the secretory process downstream from calcium influx in dopaminergic neurons: implication of K+ channels. J. Neurophysiol., 87, 1046-1056.

Cotrina, M.L., Lin, J.H., Lopez-Garcia, J.C., Naus, C.C. \& Nedergaard, M. (2000) ATP-mediated glia signaling. J. Neurosci., 20, 2835-2844.

D'Amore, P.A. (1990) Modes of FGF release in vivo and in vitro. Cancer Metastasis Rev., 9, 227-238.

Druhan, J.P. \& Wilent, W.B. (1999) Effects of the competitive $N$-methyl-Daspartate receptor antagonist, CPP, on the development and expression of conditioned hyperactivity and sensitization induced by cocaine. Behav. Brain Res., 102, 195-210.

Eclancher, F., Kehrli, P., Labourdette, G. \& Sensenbrenner, M. (1996a) Basic fibroblast growth factor (bFGF) injection activates the glial reaction in the injured adult rat brain. Brain Res., 737, 201-214.

Eclancher, F., Kehrli, P., Labourdette, G. \& Sensenbrenner, M. (1996b) Basic fibroblast growth factor (bFGF) injection activates the glial reaction in the injured adult rat brain. Brain Res., 737, 201-214.

Eclancher, F., Perraud, F., Faltin, J., Labourdette, G. \& Sensenbrenner, M. (1990) Reactive astrogliosis after basic fibroblast growth factor (bFGF) injection in injured neonatal rat brain. Glia, 3, 502-509.

Feng, L., Wang, C.Y., Jiang, H., Oho, C., Mizuno, K., Dugich-Djordjevic, M. \& Lu, B. (1999) Differential effects of GDNF and BDNF on cultured ventral mesencephalic neurons. Brain Res. Mol. Brain Res., 66, 62-70.

Flores, C., Rodaros, D. \& Stewart, J. (1998) Long-lasting induction of astrocytic basic fibroblast growth factor by repeated injections of amphetamine: blockade by concurrent treatment with a glutamate antagonist. J. Neurosci., 18, 9547-9555.
Flores, C., Salmaso, N., Cain, S., Rodaros, D. \& Stewart, J. (1999) Ovariectomy of adult rats leads to increased expression of astrocytic basic fibroblast growth factor in the ventral tegmental area and in dopaminergic projection regions of the entorhinal and prefrontal cortex. J. Neurosci., 19, $8665-8673$.

Flores, C., Samaha, A.N. \& Stewart, J. (2000) Requirement of endogenous basic fibroblast growth factor for sensitization to amphetamine. J. Neurosci., 20, RC55.

Flores, C. \& Stewart, J. (2000b) Basic fibroblast growth factor as a mediator of the effects of glutamate in the development of long-lasting sensitization to stimulant drugs: studies in the rat. Psychopharmacology (Berl), 151, 152165.

Florkiewicz, R.Z., Baird, A. \& Gonzalez, A.M. (1991) Multiple forms of bFGF: differential nuclear and cell surface localization. Growth Factors, 4, $265-275$.

Florkiewicz, R.Z., Majack, R.A., Buechler, R.D. \& Florkiewicz, E. (1995) Quantitative export of FGF-2 occurs through an alternative, energydependent, non-ER/Golgi pathway. J. Cell Physiol., 162, 388-399.

Giordano, S., Sherman, L., Lyman, W. \& Morrison, R. (1992) Multiple molecular weight forms of basic fibroblast growth factor are developmentally regulated in the central nervous system. Dev. Biol., 152, 293-303.

Haydon, P.G. (2001) GLIA: listening and talking to the synapse. Nature Rev. Neurosci., 2, 185-193.

Hortala, M., Estival, A., Pradayrol, L., Susini, C. \& Clemente, F. (2005) Identification of c-Jun as a critical mediator for the intracrine $24 \mathrm{kDa}$ FGF-2 isoform-induced cell proliferation. Int. J. Cancer, 114, 863-869.

Hosli, E. \& Hosli, L. (1993) Receptors for neurotransmitters on astrocytes in the mammalian central nervous system. Prog. Neurobiol., 40, 477-506.

Hou, J.G., Cohen, G. \& Mytilineou, C. (1997) Basic fibroblast growth factor stimulation of glial cells protects dopamine neurons from 6-hydroxydopamine toxicity: involvement of the glutathione system. J. Neurochem., 69, 76-83.

Hu, Y., Fang, X., Dunham, S.M., Prada, C., Stachowiak, E.K. \& Stachowiak, M.K. (2004) $90-\mathrm{kDa}$ ribosomal S6 kinase is a direct target for the nuclear fibroblast growth factor receptor 1 (FGFR1): role in FGFR1 signaling. J. Biol. Chem., 279, 29325-29335.

Imamura, T., Engleka, K., Zhan, X., Tokita, Y., Forough, R., Roeder, D., Jackson, A., Maier, J.A., Hla, T. \& Maciag, T. (1990) Recovery of mitogenic activity of a growth factor mutant with a nuclear translocation sequence. Science, 249, 1567-1570.

Jomphe, C., Levesque, D. \& Trudeau, L.E. (2003) Calcium-dependent, D2 receptor-independent induction of c-fos by haloperidol in dopamine neurons. Naunyn Schmiedebergs Arch. Pharmacol., 367, 480-489.

Joy, A., Moffett, J., Neary, K., Mordechai, E., Stachowiak, E.K., Coons, S., Rankin-Shapiro, J., Florkiewicz, R.Z. \& Stachowiak, M.K. (1997) Nuclear accumulation of FGF-2 is associated with proliferation of human astrocytes and glioma cells. Oncogene, 14, 171-183.

Kahlig, K.M., Binda, F., Khoshbouei, H., Blakely, R.D., McMahon, D.G., Javitch, J.A. \& Galli, A. (2005) Amphetamine induces dopamine efflux through a dopamine transporter channel. Proc. Natl Acad. Sci. USA, 102, 3495-3500.

Kalivas, P.W. \& Stewart, J. (1991) Dopamine transmission in the initiation and expression of drug- and stress-induced sensitization of motor activity. Brain Res. Brain Res. Rev., 16, 223-244.

Kalivas, P.W. \& Weber, B. (1988) Amphetamine injection into the ventral mesencephalon sensitizes rats to peripheral amphetamine and cocaine. J. Pharmacol. Exp. Ther, 245, 1095-1102.

Karler, R., Calder, L.D., Chaudhry, I.A. \& Turkanis, S.A. (1989) Blockade of 'reverse tolerance' to cocaine and amphetamine by MK-801. Life Sci., 45, 599-606.

Karler, R., Calder, L.D. \& Turkanis, S.A. (1991) DNQX blockade of amphetamine behavioral sensitization. Brain Res., 552, 295-300.

Karler, R., Chaudhry, I.A., Calder, L.D. \& Turkanis, S.A. (1990) Amphetamine behavioral sensitization and the excitatory amino acids. Brain Res., 537, 76-82.

Klagsbrun, M. \& Vlodavsky, I. (1988) Biosynthesis and storage of basic fibroblast growth factor (bFGF) by endothelial cells: implication for the mechanism of action of angiogenesis. Prog. Clin. Biol. Res., 266, $55-61$.

Klein, S., Morimoto, T. \& Rifkin, D.B. (1996) Characterization of fibroblast growth factor-2 binding to ribosomes. Growth Factors, 13, 219-228.

$\mathrm{Ku}$, P.T. \& D'Amore, P.A. (1995) Regulation of basic fibroblast growth factor (bFGF) gene and protein expression following its release from sublethally injured endothelial cells. J. Cell Biochem., 58, 328-343. 
Le, R. \& Esquenazi, S. (2002) Astrocytes mediate cerebral cortical neuronal axon and dendrite growth, in part, by release of fibroblast growth factor. Neurol. Res., 24, 81-92.

Li, Y., Hu, X.T., Berney, T.G., Vartanian, A.J., Stine, C.D., Wolf, M.E. \& White, F.J. (1999) Both glutamate receptor antagonists and prefrontal cortex lesions prevent induction of cocaine sensitization and associated neuroadaptations. Synapse, 34, 169-180.

Li, Y., Vartanian, A.J., White, F.J., Xue, C.J. \& Wolf, M.E. (1997) Effects of the AMPA receptor antagonist NBQX on the development and expression of behavioral sensitization to cocaine and amphetamine. Psychopharmacology (Berl.), 134, 266-276.

Logan, A. (1990) Intracrine regulation at the nucleus - a further mechanism of growth factor activity? J. Endocrinol., 125, 339-343.

Luo, Y., Kokkonen, G.C., Wang, X., Neve, K.A. \& Roth, G.S. (1998) D2 dopamine receptors stimulate mitogenesis through pertussis toxin-sensitive $\mathrm{G}$ proteins and Ras-involved ERK and SAP/JNK pathways in rat C6-D2L glioma cells. J. Neurochem., 71, 980-990.

McNeil, P.L., Muthukrishnan, L., Warder, E. \& D'Amore, P.A. (1989) Growth factors are released by mechanically wounded endothelial cells. J. Cell Biol., 109, 811-822.

Mignatti, P., Morimoto, T. \& Rifkin, D.B. (1992) Basic fibroblast growth factor, a protein devoid of secretory signal sequence, is released by cells via a pathway independent of the endoplasmic reticulum-Golgi complex. J. Cell Physiol., 151, 81-93.

Moffett, J., Kratz, E., Florkiewicz, R. \& Stachowiak, M.K. (1996) Promoter regions involved in density-dependent regulation of basic fibroblast growth factor gene expression in human astrocytic cells. Proc. Natl Acad. Sci. USA, 93, 2470-2475.

Mueller, D., Chapman, C. \& Stewart, J. (2006) Amphetamine induces dendritic growth in ventral tegmental area dopaminergic neurons in vivo via basic fibroblast growth factor. Neuroscience, 137, 727-735.

Nakanishi, Y., Kihara, K., Mizuno, K., Masamune, Y., Yoshitake, Y. \& Nishikawa, K. (1992) Direct effect of basic fibroblast growth factor on gene transcription in a cell-free system. Proc. Natl Acad. Sci. USA, 89, 52165220

Powell, P.P. \& Klagsbrun, M. (1991) Three forms of rat basic fibroblast growth factor are made from a single mRNA and localize to the nucleus. J. Cell Physiol., 148, 202-210.

Reilly, J.F., Maher, P.A. \& Kumari, V.G. (1998) Regulation of astrocyte GFAP expression by TGF-beta1 and FGF-2. Glia, 22, 202-210.

Renko, M., Quarto, N., Morimoto, T. \& Rifkin, D.B. (1990) Nuclear and cytoplasmic localization of different basic fibroblast growth factor species. J. Cell Physiol., 144, 108-114.

Rifkin, D.B., Quarto, N., Mignatti, P., Bizik, J. \& Moscatelli, D. (1991) New observations on the intracellular localization and release of bFGF. Ann. N.Y. Acad. Sci., 638, 204-206.

Robinson, T.E. \& Becker, J.B. (1986) Enduring changes in brain and behavior produced by chronic amphetamine administration: a review and evaluation of animal models of amphetamine psychosis. Brain Res., 396, 157-198.

Robinson, T.E. \& Berridge, K.C. (1993) The neural basis of drug craving: an incentive-sensitization theory of addiction. Brain Res. Brain Res. Rev., 18, 247-291.

Salik, E., Ercan, F., Sirvanci, S., Cetinel, S., Onat, F. \& San, T. (2005) Effect of aging on the distribution of basic fibroblast growth factor immunoreactive cells in the rat hippocampus. Brain Res. Bull., 64, 409-415.

Schweigerer, L., Neufeld, G., Friedman, J., Abraham, J.A., Fiddes, J.C. \& Gospodarowicz, D. (1987) Capillary endothelial cells express basic fibroblast growth factor, a mitogen that promotes their own growth. Nature, 325, 257 259.

Segal \& Schuckit (1983) Animals models of stimulant-induced psychosis. In Creese, I., (Ed), Stimulants: neurochemical, behavioral and clinical perspectives. Raven, New York, pp. 131-167.

Seiden, L.S., Sabol, K.E. \& Ricaurte, G.A. (1993) Amphetamine: effects on catecholamine systems and behavior. Annu. Rev. Pharmacol. Toxicol., 33, 639-677.

Sherman, L., Stocker, K.M., Morrison, R. \& Ciment, G. (1993) Basic fibroblast growth factor (bFGF) acts intracellularly to cause the transdifferentiation of avian neural crest-derived Schwann cell precursors into melanocytes. Development, 118, 1313-1326.

Stachowiak, E.K., Maher, P.A., Tucholski, J., Mordechai, E., Joy, A., Moffett, J., Coons, S. \& Stachowiak, M.K. (1997a) Nuclear accumulation of fibroblast growth factor receptors in human glial cells - association with cell proliferation. Oncogene, 14, 2201-2211.
Stachowiak, M.K., Moffett, J., Joy, A., Puchacz, E., Florkiewicz, R. \& Stachowiak, E.K. (1994) Regulation of bFGF gene expression and subcellular distribution of bFGF protein in adrenal medullary cells. J. Cell Biol., 127, 203-223.

Stachowiak, M.K., Moffett, J., Maher, P., Tucholski, J. \& Stachowiak, E.K. (1997b) Growth factor regulation of cell growth and proliferation in the nervous system. A new intracrine nuclear mechanism. Mol. Neurobiol., 15, $257-283$.

Stewart, J. \& Druhan, J.P. (1993) Development of both conditioning and sensitization of the behavioral activating effects of amphetamine is blocked by the non-competitive NMDA receptor antagonist, MK-801. Psychopharmacology (Berl), 110, 125-132.

St-Gelais, F., Legault, M., Bourque, M.J., Rompre, P.P. \& Trudeau, L.E. (2004) Role of calcium in neurotensin-evoked enhancement in firing in mesencephalic dopamine neurons. J. Neurosci., 24, 2566-2574.

Sulzer, D., Chen, T.K., Lau, Y.Y., Kristensen, H., Rayport, S. \& Ewing, A. (1995) Amphetamine redistributes dopamine from synaptic vesicles to the cytosol and promotes reverse transport. J. Neurosci., 15, 41024108.

Sulzer, D., Joyce, M.P., Lin, L., Geldwert, D., Haber, S.N., Hattori, T. \& Rayport, S. (1998) Dopamine neurons make glutamatergic synapses in vitro. J. Neurosci., 18, 4588-4602.

Szele, F.G., Alexander, C. \& Chesselet, M.F. (1995) Expression of molecules associated with neuronal plasticity in the striatum after aspiration and thermocoagulatory lesions of the cerebral cortex in adult rats. J. Neurosci., 15, 4429-4448.

Tessler, S. \& Neufeld, G. (1990) Basic fibroblast growth factor accumulates in the nuclei of various bFGF-producing cell types. J. Cell Physiol., 145, 310 317.

Trudel, C., Faure-Desire, V., Florkiewicz, R.Z. \& Baird, A. (2000) Translocation of FGF2 to the cell surface without release into conditioned media. J. Cell Physiol., 185, 260-268.

Ullian, E.M., Christopherson, K.S. \& Barres, B.A. (2004) Role for glia in synaptogenesis. Glia, 47, 209-216.

Vezina, P. (1993) Amphetamine injected into the ventral tegmental area sensitizes the nucleus accumbens dopaminergic response to systemic amphetamine: an in vivo microdialysis study in the rat. Brain Res., 605, 332-337.

Vezina, P. (1996) D1 dopamine receptor activation is necessary for the induction of sensitization by amphetamine in the ventral tegmental area. J. Neurosci., 16, 2411-2420.

Vezina, P., Lorrain, D.S., Arnold, G.M., Austin, J.D. \& Suto, N. (2002) Sensitization of midbrain dopamine neuron reactivity promotes the pursuit of amphetamine. J. Neurosci., 22, 4654-4662.

Vezina, P. \& Stewart, J. (1990) Amphetamine administered to the ventral tegmental area but not to the nucleus accumbens sensitizes rats to systemic morphine: lack of conditioned effects. Brain Res., 516, 99-106.

Vlodavsky, I., Fridman, R., Sullivan, R., Sasse, J. \& Klagsbrun, M. (1987) Aortic endothelial cells synthesize basic fibroblast growth factor which remains cell associated and platelet-derived growth factor-like protein which is secreted. J. Cell Physiol., 131, 402-408.

Wiedlocha, A., Falnes, P.O., Madshus, I.H., Sandvig, K. \& Olsnes, S. (1994) Dual mode of signal transduction by externally added acidic fibroblast growth factor. Cell, 76, 1039-1051.

Williams, E.J., Mittal, B., Walsh, F.S. \& Doherty, P. (1995) FGF inhibits neurite outgrowth over monolayers of astrocytes and fibroblasts expressing transfected cell adhesion molecules. J. Cell Sci., 108, 3523-3530.

Wolf, M.E. \& Xue, C.J. (1998) Amphetamine and D1 dopamine receptor agonists produce biphasic effects on glutamate efflux in rat ventral tegmental area: modification by repeated amphetamine administration. J. Neurochem., 70, 198-209.

Wolf, M.E. \& Xue, C.J. (1999) Amphetamine-induced glutamate efflux in the rat ventral tegmental area is prevented by MK-801, SCH 23390, and ibotenic acid lesions of the prefrontal cortex. J. Neurochem., 73, 1529-1538.

Wolf, M.E., Xue, C.J., Li, Y. \& Wavak, D. (2000) Amphetamine increases glutamate efflux in the rat ventral tegmental area by a mechanism involving glutamate transporters and reactive oxygen species. J. Neurochem., 75, 1634-1644.

Woodward, W.R., Nishi, R., Meshul, C.K., Williams, T.E., Coulombe, M. \& Eckenstein, F.P. (1992) Nuclear and cytoplasmic localization of basic fibroblast growth factor in astrocytes and CA2 hippocampal neurons. J. Neurosci., 12, 142-152.

Zanassi, P., Paolillo, M., Montecucco, A., Avvedimento, E.V. \& Schinelli, S. (1999) Pharmacological and molecular evidence for dopamine $\mathrm{D}(1)$ receptor expression by striatal astrocytes in culture. J. Neurosci. Res., 58, 544-552. 